\title{
A Queuing Approach for Energy Supply in Manufacturing Facilities
}

\author{
Lucio Zavanella, Ivan Ferretti, Simone Zanoni, and Laura Bettoni \\ Department of Mechanical and Industrial Engineering \\ Università degli Studi di Brescia, \\ via Branze, 38, I-25123, Brescia, Italy \\ \{zavanell, ivan. ferretti, zanoni, laura.bettoni\} @ing.unibs.it
}

\begin{abstract}
Nowadays increasing energy efficiency is one of the main objectives of manufacturing systems so as to remain competitive despite of the foreseen increase in energy prices for the next years. We propose a novel queuing-based model for the appreciation of the energy consumption on a company base, so as to optimize the total energy costs due to electricity utilization.

We propose an analytical model based on the extension of the $\mathrm{M}^{[\mathrm{x}]} / \mathrm{M} / \infty$ model where arrivals of the queuing model represent the statistical distribution of switch-on of a generic set of machines and departures represent statistical distribution of the resources switch-off. This model can be easily used to assess and establish the contract with the energy supplier under optimal parameters of contractual power, based on its tariff components. Numerical examples are offered to show the applicability of the proposed model.
\end{abstract}

Keywords: energy efficiency, Energy-aware models.

\section{$1 \quad$ Introduction}

Manufacturing systems are usually organized in multiple departments and in many of them there are multiple machines and each machine has its own electricity demand pattern over time. This energy requirement is usually different from machine to machine and it depends on the power of the machine and on the relative duration of the different states (idle, standby, load level, machining parameters, maximum speed) determined by different product routings which encompass it. The practical problem lies on the fact that a company does not have access to endless amounts of electricity, or that it is already subject to a contract with the supplier, which generally provides a maximum level of supply which, when exceeded, determine substantial penalties to the user.

For this reason, the objective is to create a model, of simple use, able to assist energy managers who, thanks to the model itself, may simulate different production scenarios under different electricity supply contracts, so as to minimize the expected costs. In particular, the application of the model may lead to the calculation of the following performance indices: 
- probability of exceeding a specific level of power requirement;

- expected average-power requirement;

- economic evaluation and comparison of different electricity supply contracts.

Dietmair and Verl [1] introduced a generic method so as to model the energy consumption behavior of machines based on a statistical discrete event formulation. The parameter information, required to characterize the discrete events, can be obtained by a small number of simple measurements or by a degree of uncertainty from the machine and component documentation.

Bruzzone et al. [2] proposed the integration of an EAS module (energy-aware scheduling), within an advanced planning and scheduling (APS) system, incorporating a model to control the shop-floor power peak for a given detailed schedule; unavailability of the actual machine tool power profile and idle-state energy consumption require to assume constant mean power demand for each job. The goal of EAS is to optimize the given schedule from the viewpoint of the energy consumption, while keeping the given assignment and sequencing fixed. The problem is discussed by minimizing the shop-floor power peak, while limiting the possible worsening of the two scheduling objectives (tardiness and makespan minimization), modeling it as a Mixed Integer Programming (MIP) problem.

He et al. [3] proposed a modeling method of task-oriented energy consumption for machining manufacturing system. The energy consumption characteristics, driven by task flow in machining manufacturing system, are analyzed, thus describing how energy consumption dynamically depends on the flexibility and variability of task flow in production processes. The results show a valuable insight of energy consumption in machining manufacturing system, so as to make robust decisions on the potential for improving energy efficiency.

Prabhu et al. [4] proposed a queuing models to predict energy savings in serial production lines, where idling machines are switched to a lower power state in serial production lines consisting of machines with Poisson arrival and exponential service time.

Prabhu and Jeon [5] extended their previous model and generalize the energy aware queuing model to a re-entrant structure. Moreover, an application to a semiconductor factory is presented.

The paper is organized as follows: section 2 presents the system and the main assumptions. In Section 3, a model and the energy cost function are presented. In Section 4 a numerical example is offered. Section 5 summarizes the paper content, describing possible future research directions.

\section{The System}

One of the most important managerial actions to reduce energy costs is the proper fitting of the energy supply contracts with the plant requirements. In particular, these contracts generally provide the maximum thresholds that, when exceeded, require the payment of penalties. For example, in the electricity contract there is a threshold for the maximum power contemporary required by the loads, also defined as "contractual power". The problem faced in this paper is to determine the appropriate contractual power considering the variability in electricity usage (given by stochastic distribution 
of loads switch on and switch off), which is generally not predictable a priori. A simple model is then proposed, derived from queuing theory, which allows to model adequately the variability of the electrical energy consumption and to suggest the contractual power more correct, adopting a probabilistic approach to the economic optimization.

Queuing theory is capable of providing models (and consequent insights) able to predict the system behavior when jobs, i.e. system customers, ask for providing services at a randomly occurring demand. In a queuing model, entities (data, parts, jobs, etc.) arrive at the system and require for some form of service (operations, machining, assembly processes, etc.). Due to demand variability and service capability, a queue is formed. The idea is to model the variability in electricity usage by a queuing system, where the entities are represented by the power devices of the department considered. In particular, the distribution of arrivals describes the devices activation, while the distribution of services describes their shutdown. Given the random nature of the two distributions, two exponential distributions may be considered. Obviously, this assumption is valid in departments where the consumption of these devices is not easy to be predicted. Thus, the arrival and the service rates of the queuing system are expressed as power per unit of time. In such a queuing system, the average number of entities in the system is a strategic information to assess the contractual power. The queuing system defined is depicted in the following figure.

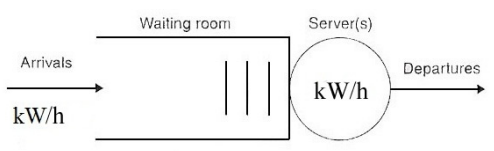

Fig. 1. The system considered in the analysis

In order to obtain tractable models, it is assumed that the arrival process and the service process are stationary. Figure 2 shows how the electric power required by a generic machine is variable (for reference see $\mathrm{He}$ et al, 2012) because, in general, power varies with respect to the states in which the machine is (e.g., state of heating, pressing state, stand-by). In order to simplify the problem, it may be useful to define the required power as constant, as shown below. In the next section the queuing model proposed is presented.
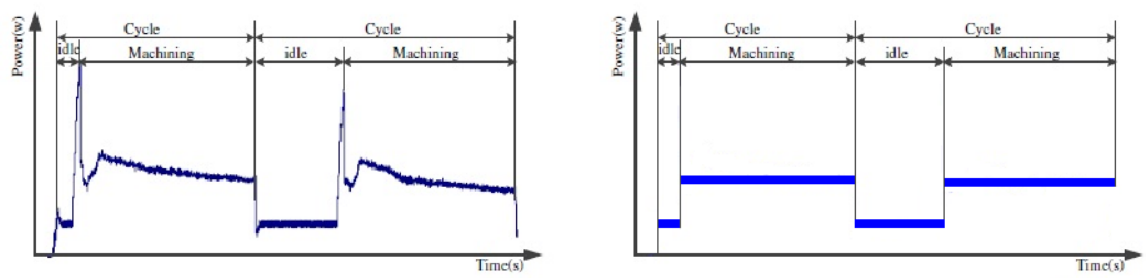

Fig. 2. Variation of the required power according to the machine states 


\section{Model}

The model of the queuing theory proposed for the solution of the problem investigated is $M^{[x]} / M / \infty$. In particular, we assume, in addition to the assumption that the arrival stream fits a Poisson process, that the actual number of entities in any arriving module is a random variable $X$, which takes on the value $k$ with probability $w_{k}$, where $k$ is a positive integer. Because the Poisson process models the arrival of one - and only one - entity per arrival event, we consider a bulk input. This assumption models the simultaneous activation of multiple devices or the activation of a device at different power levels.

Moreover, we assume infinite service capacity, because every device is activated instantly (i.e., no queue is allowed).

After the definition of the birth-death process and the equations governing the system, using a generating function approach (Gross and al., 2008), it is possible to calculate the average number of entities in the system $L=\sum_{i=0}^{X}\left(i \cdot p_{i}\right)$. This performance index is the average power required. Following, we show the rate balance equations related to the states $0-1$ and the states $1-2$ :

$$
\begin{gathered}
\lambda \sum_{k=1}^{X} w_{k} p_{0}=\mu p_{1} \quad \rightarrow \quad p_{1}=\frac{\lambda}{\mu} p_{0} \\
\lambda \sum_{k=1}^{X} w_{k} p_{1}+\lambda\left(1-w_{1}\right) p_{0}=\mu p_{2}
\end{gathered}
$$

Using the Little's law and assuming infinite service capacity, the average waiting time in the system is:

$$
W=\frac{1}{\mu}
$$

In the industrial context, the power required by the department, or company, is always satisfied by the supplier of the electric power, even if the power required exceeds the predetermined contractual power. Whenever the power required exceeds the contractual threshold, the consumer will be subjected to a penalty. The objective of the analysis proposed is to verify in which cases it may be convenient to pay penalties instead of having a greater contractual power and vice versa.

In order to compare the cost of energy, in case of exceeding or not the power threshold, the following objective function is defined. The model is not based on energy consumption, but on the probability of being in a given state, thus requiring power at a given level. Thus, the following formulae will be based on these probability distributions.

In particular, the objective function (expressed as a rate $€ / h$ ) is composed by three parts: the first is the evaluation of the cost below the threshold, while the second is the evaluation of the cost above the threshold and the third part is the cost proportional to the threshold chosen: 


$$
\operatorname{EEC}(P)=\sum_{i=0}^{P} p_{i} \cdot \alpha \cdot i+\sum_{i=P+1}^{P \max } p_{i} \cdot[\alpha \cdot P+\beta \cdot(i-P)]+\gamma \cdot P
$$

where:

- $\quad \alpha$ is the energy cost below the threshold [€/kWh]

- $\quad \beta$ is the energy cost above the threshold [€/kWh]

- $\quad \gamma$ is the energy cost related to the threshold $[(€ / \mathrm{kW})(1 / \mathrm{h})]$

- $\quad P$ is the threshold (contractual power supply) $[\mathrm{kW}]$

- $\quad P_{\text {max }}$ is the maximum power beyond the threshold $[\mathrm{kW}]$

- $\quad p_{i}$ is the probability of requiring a power level equal to $i$

\section{$4 \quad$ Numerical Analysis}

So as to study the behavior of the model, a numerical analysis was carried out to investigate how the model parameters influence the optimal solution of the energy model. The scenario considered consists of a production system with machine tools with a required power that can be represented by four different batch sizes with linearly increasing probability distribution. The arrival rate is equal to $10 \mathrm{~kW} / \mathrm{h}$ (e.g., 10 events of $1 \mathrm{~kW}$ per hour), while the service rate is $35 \mathrm{~kW} / \mathrm{h}$ (the arrival and service rates have been obtained by a fitting analysis of the statistical data of the real profile of power consumption in a basic shop floor).

Given a particular energy supply contract with $\alpha=0.2[€ / \mathrm{kWh}], \beta=0.3[€ / \mathrm{kWh}]$, $\gamma=0.05[(€ / \mathrm{kW})(1 / \mathrm{h})]$, the average power required is equal to $12.99 \mathrm{~kW}$. Thus, a first option could be to set a contractual power at 13 [kW]: according to the formulae listed above, the expected hourly energy cost is equal to $3.77[€ / \mathrm{h}]$.

Alternative options could be to adopt a supply contract larger than the average, e.g. $20 \mathrm{~kW}$, or a supply contract smaller than the average, e.g. $10 \mathrm{~kW}$.

According to the formulae shown in the previous section, the expected hourly energy cost for the supply contract with $20 \mathrm{~kW}$ is equal to $3.92[€ / \mathrm{h}]$, while for the supply contract with $10 \mathrm{~kW}$ is equal to $3.74[€ / \mathrm{h}]$. Thus, in this case it is preferable to operate with a smaller power contract and pay the penalties, due to the probability of exceeding the contract power.

\section{Conclusion}

This work faced the problem of the electrical energy supply contract in an industrial environment. The main objective is to properly adjust the contractual power in a manufacturing system composed by several electrical resources and analyze the power consumption probability distribution applying queuing theory. The electrical power demand has been viewed as a physical user (client) that requests access to a system (electrical network) for a certain period of time (thus resulting in the consumption). 
The mathematical model of a $M^{[x]} / M / \infty$ queue fits this case and it helps in capturing the dynamics of the system. Moreover, using synthetic formulae with probability functions it is possible to look for the most convenient electrical supply contract, given a set of offer available from the suppliers.

\section{References}

1. Dietmair, A., Verl, A.: A generic energy consumption model for decision making and energy efficiency optimisation in manufacturing. International Journal of Sustainable Engineering 2(2), 123-133 (2009)

2. Bruzzone, A.A.G., Anghinolfi, D., Paolucci, M., Tonelli, F.: Energy-aware scheduling for improving manufacturing process sustainability: A mathematical model for flexible flow shops. CIRP Annals - Manufacturing Technology 61(1), 459-462 (2012)

3. He, Y., Bo Liu, B., Zhang, X., Gao, H., Liu, X.: A modeling method of task-oriented energy consumption for machining manufacturing system. Journal of Cleaner Production 23(1), 167-174 (2012)

4. Prabhu, V.V., Jeon, H.W., Taisch, M.: Modeling Green Factory Physics - An Analytical Approach. In: 8th IEEE International Conference on Automation Science and Engineering, Seoul, Korea, August 20-24 (2012)

5. Jeon, H.W., Prabhu, V.V.: Modeling Green Fabs - A Queuing Theory Approach for Evaluating Energy Performance. In: Emmanouilidis, C., Taisch, M., Kiritsis, D. (eds.) APMS 2013. IFIP AICT, vol. 397, pp. 41-48. Springer, Heidelberg (2013) 